\title{
Doing outreach with the first direct observation of gravitational waves
}

\author{
Nicolas Arnaud*, for the Virgo Collaboration and the LIGO Scientific Collaboration \\ Laboratoire de l'Accélérateur Linéaire, Univ. Paris-Sud, CNRS/IN2P3, Orsay, France \\ European Gravitational Observatory, Cascina, Italy \\ E-mail: narnaudelal.in2p3.fr
}

\begin{abstract}
On February 11 2016, the LIGO Scientific Collaboration and the Virgo Collaboration announced the observation of gravitational waves from a binary black hole merger. The transient signal GW150914 had been recorded five months earlier by the two Advanced LIGO detectors which had just started their first observation run, following a few years of upgrade. Parallel to the data analysis which led to the conclusion that the observed signal was indeed of astrophysical origin, the two collaborations invested a lot of effort to prepare the discovery announcement. In these proceedings, we review the main aspects of the outreach strategy developed for this event and try to quantify its impact on the various targeted audiences: the general audience, the media and the educational community.
\end{abstract}

38th International Conference on High Energy Physics

3-10 August 2016

Chicago, USA

\footnotetext{
* Speaker.
} 


\section{The first two direct detections of gravitational waves}

In February and June 2016, the LIGO Scientific Collaboration and the Virgo Collaboration announced the first two direct detections of gravitational waves (GW). Both events - GW150914 [1] (observed on September 15 2015) and GW151226 [2] (observed on December 26 2016) - are binary black hole $(\mathrm{BBH})$ coalescences and have been recorded by the two Advanced LIGO (aLIGO) detectors [3]. These events trigger the dawn of GW astronomy as they are the first direct detections of black holes (BH): so far BHs had only been seen indirectly, through their gravitational effect on neighboring stars. These also are the first directions of black holes in the mass range of a few tens of Solar masses and the first detections of BBHs. More details about these discoveries can be found in the literature and in various plenary and parallel talks given at this conference.

\section{Discovery announcement}

It only took a few minutes for the online data analysis software to identify GW150914 as an interesting event and less than a day for the LIGO and Virgo collaborations to realize that this was a strong $\mathrm{BBH}$ candidate recorded at a time when no known problem was affecting the aLIGO detectors. Yet, the discovery was only announced five months later, on Thursday February 11 2016. In the meantime, extensive data analysis was carried out to make the detection case rocksolid. Parallel to this, communication offices and LIGO-Virgo outreach groups were preparing the announcement [4] together with all the players involved: observatories, scientific collaborations, funding agencies from the various partner countries (USA, UK, Germany, France, Italy to name a few), laboratories and universities. During the whole process, the potential discovery had to be kept secret, that is internal to LIGO and Virgo. Indeed, any data analysis step or data quality check could potentially turn the promising GW event into a false alarm. With about 1,000 people involved in the process or at least aware of the existence of a strong GW candidate, leaks could have become an issue. In case of questions coming from outside the collaborations, a simple (and, by the way, true) answer had been prepared: "the analysis of the data taken during the first Observation Run (O1) of the aLIGO detectors is in progress; results will be made public when the analysis is complete". In the end, some limited information leaked outside LIGO-Virgo and a few people tried to exploit it, mainly on social media. But this was only a minor annoyance; the physics analysis and the communication \& outreach plan could proceed as planned. One of the main complications for what concerned communication and outreach was the need to target simultaneously various different audiences: first the scientific community (the GW discovery is the completion of a century-long quest), then the general public with basic scientific knowledge and finally high-school teachers. Regarding the latter point, a wide set of educational resources was developed, from basic schematics and analogies, to science summaries of the main scientific publication. In addition, emphasis was set on social media (Twitter, Facebook and Reddit) to promote the scientific results and the associated education \& outreach $(\mathrm{E} \& \mathrm{O})$ projects. During the whole process of the discovery announcement preparation, a key reference was the announcement of the H boson discovery by the LHC experiments ATLAS and CMS at CERN [5].

The strategy selected for the discovery announcement was the following. First to write a discovery article and to have it accepted by the targeted journal (Physics Review Letters) prior to 
announcing the discovery. That article was to be supported by several "companion papers" [6], each focusing on a particular aspect of the global analysis (instruments, data quality, astrophysical implications of the discovery, etc.). As for the day of the announcement, a promising date was Thursday February 11 2016, the first day of the AAAS ("American Association for the Advancement of Science") annual meeting to be held in Washington DC. Therefore, we had some sort of internal countdown to drive the progress of the internal review of the analysis, such as the development of the communication and $\mathrm{E} \& \mathrm{O}$ plans. In the end, this helped to make sure that all these parallel projects would converge simultaneously and would not take too long - keeping the discovery information secret was getting more and more challenging as time was going. It was decided to have a simultaneous and worldwide announcement of the discovery with two main press conferences: Washington DC (LIGO, AAAS meeting) and Cascina (in the European Gravitational Observatory, EGO, the site of the Virgo detector). In addition, several satellite press briefings were to be organized, for instance at the CNRS headquarters in Paris. The time of the announcement was set as 10:30 EST, a compromise given the various timezones involved. It was quite early on the West Coast (07:30 local time) and quite late in Europe (16:30 in Paris for instance) to have the discovery covered by the evening news channels. The press releases were coordinated. They all included the exact same compulsory parts; in particular, they shared a common opening section. They could contain additional text, written at the discretion of the organization issuing that particular press release. And they were translated into 18 languages, including Siksika, which is the language of the Blackfoot Indians in Canada and the US. The science summaries [7] written to explain the discoveries to a broad audience were multilingual as well.

The discovery made its way to the Newseum front page archives [8] where it is one of the few positive events listed for 2016. 961 front pages featuring the discovery on February 12 were listed by the website. More than 200 journalists followed the announcement, either in person or through webcasts. There were about 5,000 related press articles in the world, ranging from daily newspapers to magazines. And more than 500,000 Internet users watched the webcasts, either live or at a later time. On Twitter, the hashtags \#gravitationalwaves, \#LIGO and \#EinsteinWasRight had more than 70 million impressions. And for instance there were 10 million impressions for the equivalent hashtag in French, \#ondesgravitationnelles. The number of followers of the experiment Twitter accounts increased significantly: by more than a factor 10 for @ego_virgo, from 3,000 to more than 25,000 (after the second discovery announcement) for @ ligo. And there were mentions, retweets or likes from celebrities - e.g. @ POTUS (acronym for "President of the United States"). On Facebook, the experiments got new followers: 9,000 for LIGO, 1,000 for Virgo. AMA ("Ask Me Anything") Reddit live sessions were organized following both announcements and had a large attendance. Moreover, GW150914 appeared on the Astronomy Picture of the Day webpage [9] which had more than 1 million views in the week following the announcement. The first discovery was also transmitted through well-known online scientific medias, like "Minute Physics" [10] and "PhD Comics" [11].

\section{Outreach resources and activities}

The LIGO-Virgo collaboration and many individual members have developed a variety of $\mathrm{E} \& \mathrm{O}$ projects related to $\mathrm{GW}$. Some were already existing before the detections while others were 


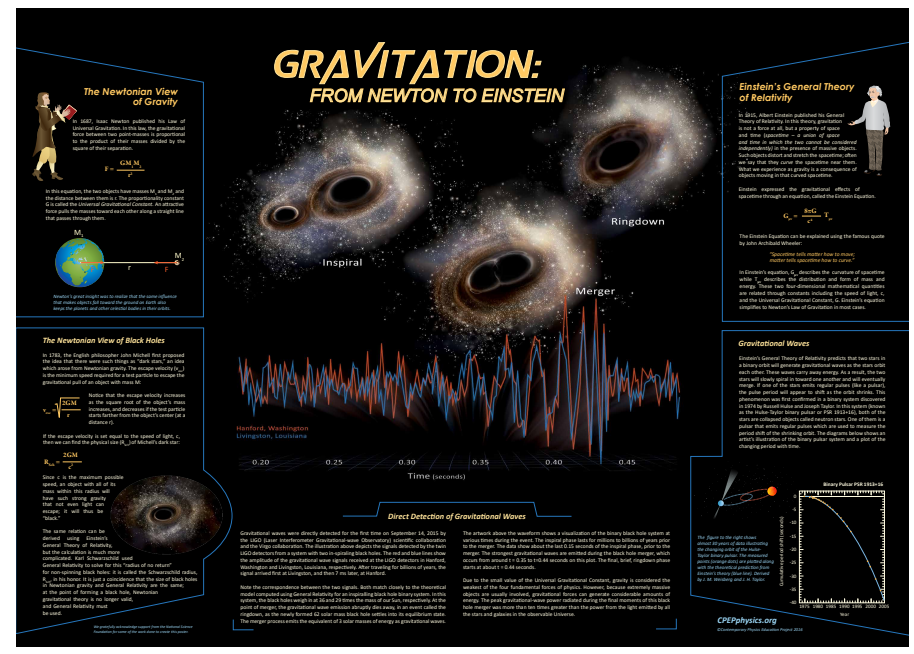

Figure 1: CPEP poster about gravitation and gravitational waves.

created to go along with them. New projects are in preparation as well. Only a sample of these efforts can be presented in these proceedings; the reader interested in knowing more is welcome to visit our websites and/or to contact the various $\mathrm{E} \& \mathrm{O}$ groups [12]. In addition, a forum has been setup to answer questions from the general audience: question@ligo.org. Among the variety of E\&O resources, the LIGO Open Science Center (LOSC) [13] is playing a key role. Similar to, for instance, the CERN open data portal [14], the LOSC is providing free access to some of the LIGO datasets. In particular, 1 hour of the GW channel " $h(t) "$ is available for both BBH events. These data were made available simultaneously with the discovery announcements. In addition, Python-based tutorials are provided to the users, with varying technical difficulties: some of them can be used to produce a set of plots in an automated way while others explain how to use the data.

Various resources are available for high-school teachers and students. Among which a new CPEP ("Contemporary Physics Education Project") poster about gravitation [15] which features the detection of GW - see Fig. 1. In addition to the English version, translations to other languages are in progress. A GW Educator's guide [16] is available; it has been updated to include the GW detection. An online course [17] is also provided each Summer by the Sonoma State University. The GW observatories [18] can also be visited although various constraints limit the number of visitors: the remote site locations, the need to limit anthropic noise close to the instruments and finally the limited staff available. Therefore, there are usually defined timeslots for visits, e.g. monthly opening days. School field trips and private tours need to be booked in advance. Finally, sites may be open for special events, e.g. the yearly European Researchers Night. Obviously, the attendances are larger and the number of visit requests has increased significantly since the discovery announcements. Therefore, projects of virtual visits - both standalone (a website) and live (connection with scientists on site, including a presentation and a Q\&A session) - are in progress. Various outreach resources are available online. In addition to the detections themselves [19], many websites focus on one aspect or the other of the search for GW. "Known Stellar Mass Black holes" [20] provides basic information for the known stellar-mass BH (both directly and indirectly observed) and allows one to visually compare their "sizes". The "Gravoscope" [21] combines electromagnetic and GW 
sky maps, while "GWplotter" [22] allows one to compare the sensitivities of different GW detectors with the expected sources in various frequency bands. Outreach information is also available through magazines [23]. In addition, a dedicated Youtube channel has been setup [24]: it provides explainer videos (about the sources of GW and the first two detections), talks, simulations, etc. Citizen science is not forgotten. The Einstein@Home project [25] has been using already for a few years the idle time of many computers to look for GW signals from pulsars - an extremely computer-intensive search. It is one of the most popular websites based on the BOINC framework. Last but not least, Gravity Spy [26], hosted on the Zooniverse portal has recently been put online. The idea is that users will help classifying instrumental noise transients by looking at the time-frequency maps of these glitches. Indeed, background identification and removal is a key task to improve the detector sensitivity to real GW signals. Finally, some serious games and apps have been developed [27]. For instance, Black Hole Hunter gives a flavor of what the search for a known GW signal buried in noise may look like. Internet users listen to audio files which contain noise and possibly some signals, which the ears need to trigger on. Depending on the GW characteristics (mainly its signal-to-noise ratio and frequency range), this is more or less easy.

\section{Outlook}

A wide range of $\mathrm{E} \& \mathrm{O}$ activities related to $\mathrm{GW}$ and $\mathrm{GW}$ detection by giant interferometric detectors have been presented in these proceedings. While many of these projects started a while ago, the first two detections did change everything. There are real data and excitement to share with the general public, teachers and students! As these proceedings are being written the second LIGO Observation Run ("O2") has started [28]. The two aLIGO detectors may be joined by the Advanced Virgo detector (AdV) next spring. More generally, the worldwide network of interferometric detectors will grow in the coming years while the sensitivity of the individual instruments will improve. More events should be detected (not only BBHs, but possibly other sources, for instance involving a neutron star) with the main challenge being to locate a GW source in the sky, possibly by doing a multi-messenger analysis (GW + electromagnetic radiation + possibly neutrinos). In 1983, Kip Thorne, one of the founders of the LIGO project, wrote: "That the quest ultimately will succeed seems almost assured. The only question is when and with how much further effort". On February 11 2016, the first words of David Reitze, director of the LIGO project were: "Ladies and Gentlemen. We have detected gravitational waves. We did it." The dawn of GW astronomy is not just a major scientific breakthrough; it is also an opportunity to disseminate science to attract future scientists to our field and to explain to citizens why basic science matters and why its projects require resources and time to reach their goals.

\section{References}

[1] LIGO Scientific and Virgo Collaborations (B.P. Abbott et al.), Observation of Gravitational Waves from a Binary Black Hole Merger, PRL 116 (2016) no.6 061102 [arXiv: 1602 . 03837].

[2] LIGO Scientific and Virgo Collaborations (B. P. Abbott et al.), GW151226: Observation of Gravitational Waves from a 22-Solar-Mass Binary Black Hole Coalescence, PRL 116 (2016) no.24 241103 [arXiv: 1606.04855$]$. 
[3] LIGO Scientific and Virgo Collaborations (B.P. Abbott et al.), GW150914: The Advanced LIGO Detectors in the Era of First Discoveries, PRL 116 (2016) no.13 131103 [arXiv: 1602 . 03838].

[4] J. Shapiro Key, M. Hendry, Defining gravity, Nature Physics Volume 12 (2016) Issue 6 pp. 524-525.

[5] http://press.cern/press-releases/2012/07/ cern-experiments-observe-particle-consistent-long-sought-higgs-boson

[6] https://www.ligo.caltech.edu/page/detection-companion-papers

[7] http://www.ligo.org/science/outreach.php

[8] http://www.newseum.org/todaysfrontpages/?tfp_display= archive-date\&tfp_archive_id=021216

[9] https://apod.nasa.gov/apod/ap160211.html

[10] https://www. youtube.com/watch?v=YHS9g72npqA

[11] http://www.phdcomics.com/comics/archive.php/comic/archive.php? comicid=1853

[12] https://www.ligo.caltech.edu/page/educational-resources (LIGO Laboratories), http://www.ligo.org/public.php (LIGO Scientific Collaboration), http://public.virgo-gw.eu/language/en (Virgo)

[13] https://losc.ligo.org/about

[14] http://opendata.cern.ch

[15] http://www. cpepphysics.org/gravitation.html

[16] http://www.cpepphysics.org/images/LIGOEdGuide_Final.pdf

[17] https://universe. sonoma.edu/cosmo/course/view.php?id=6

[18] https://www.ligo.caltech.edu/WA/page/lho-tours (LIGO Hanford), https: / / www.ligo.caltech.edu/LA/page/Tours (LIGO Livingston), http://public.virgo-gw.eu/visit (Virgo).

[19] http://ligo.org/detections.php (LIGO), https://owncloud.ego-gw.it/index.php/s/WGB7 jLJvU9tW7sF (Virgo)

[20] https://gravity.astro.cf.ac.uk/plotgw/bhbubble.html

[21] http://astrog80.astro.cf.ac.uk/Gravoscope

[22] http://rhcole.com/apps/GWplotter

[23] http://www.ligo.org/magazine (LIGO), http://www.ego-gw.it/public/hletter/hletter.aspx (Virgo)

[24] https: / / www - youtube.com/channel/UCMAT JmzibndbcdY8s9Prhjg

[25] https://www.einsteinathome.org

[26] https://www.zooniverse.org/projects/zooniverse/gravity-spy

[27] http: //www.blackholehunter.org (Black Hole Hunter), http://www.gwoptics.org/processing/space_time_quest (Space Time Quest), https: //www. laserlabs.org/stretchandsquash.php (Stretch and Squash), http://www.gwoptics.org/processing/blackhole_pong (Black Hole Pong) and https: / / www. laserlabs.org/pocketblackhole.php (Pocket Black Hole)

[28] https://www.ligo.caltech.edu/news/2016-11-30 